\title{
Consistent QFT description of non-standard neutrino interactions
}

\author{
Adam Falkowski, ${ }^{a}$ Martín González-Alonso ${ }^{b}$ and Zahra Tabrizi ${ }^{c, d}$ \\ ${ }^{a}$ Université Paris-Saclay, CNRS/IN2P3, IJCLab, \\ 91405 Orsay, France \\ ${ }^{b}$ Departament de Física Teòrica, IFIC, Universitat de València - CSIC, \\ Apt. Correus 22085, E-46071 València, Spain \\ ${ }^{c}$ Instituto de Física Gleb Wataghin, Universidade Estadual de Campinas (UNICAMP), \\ Rua Sérgio Buarque de Holanda, 77n, Campinas, SP, 13083-859, Brazil \\ ${ }^{d}$ Center for Neutrino Physics, Department of Physics, Virginia Tech, \\ Blacksburg, VA 24061, U.S.A. \\ E-mail: adam.falkowski@th.u-psud.fr, Martin.gonzalez@ific.uv.es, \\ ztabrizi@vt.edu
}

ABSTRACT: Neutrino oscillations are precision probes of new physics. Apart from neutrino masses and mixings, they are also sensitive to possible deviations of low-energy interactions between quarks and leptons from the Standard Model predictions. In this paper we develop a systematic description of such non-standard interactions (NSI) in oscillation experiments within the quantum field theory framework. We calculate the event rate and oscillation probability in the presence of general NSI, starting from the effective field theory (EFT) in which new physics modifies the flavor or Lorentz structure of charged-current interactions between leptons and quarks. We also provide the matching between the EFT Wilson coefficients and the widely used simplified quantum-mechanical approach, where new physics is encoded in a set of production and detection NSI parameters. Finally, we discuss the consistency conditions for the standard NSI approach to correctly reproduce the quantum field theory result.

Keywords: Effective Field Theories, Neutrino Physics

ArXiv EPrint: 1910.02971 


\section{Contents}

1 Introduction 1

2 Formalism $\quad 2$

2.1 QFT description 2

2.2 QM-NSI description 3

3 Matching QFT and QM-NSI results 4

3.1 SM interactions $\quad 5$

$3.2 V$ - $A$ interactions $\quad 6$

3.3 General case 6

$\begin{array}{lll}4 & \text { Application to specific processes } & 7\end{array}$

$\begin{array}{lll}5 & \text { Oscillation probability } & 10\end{array}$

6 Muon decay 12

7 Discussions and conclusions $\quad 14$

$\begin{array}{ll}\text { A Oscillations in QFT } & 15\end{array}$

\section{Introduction}

Precision measurements at low energies are sensitive probes of fundamental interactions that complement collider searches. Neutrino oscillation experiments [1] are a specific class thereof where one observes a characteristic oscillatory dependence of the neutrino detection rate as a function of the neutrino energy $E_{\nu}$ and the distance between the neutrino source and detector. The large body of oscillation data so far has established the existence of at least three distinct neutrino states with different masses [2,3], which is consistent with the predictions of the Standard Model (SM) supplemented with dimension- 5 terms leading to Majorana masses for the SM neutrinos [4]. Within this paradigm, neutrino mass squared differences and angles of the PMNS mixing matrix have been measured with good accuracy. This opens the door to also probing and constraining new physics (NP), by which we mean non-standard interactions (NSI) between neutrinos and matter that arise from physics beyond the SM (BSM) [5-24]. To this end, however, one needs a map between fundamental BSM parameters and observables in oscillation experiments. In this paper we construct such a map for the EFT of SM degrees of freedom, in which NP modifies the chargedcurrent interactions between neutrinos, charged leptons, and quarks. This map makes possible to understand the BSM implications of a given neutrino measurement, but also 
to combine and compare the obtained bounds with other probes that are sensitive to the same non-standard interactions (such as collider searches, $\mu \rightarrow e \gamma$, etc.).

We also discuss the consistency conditions for the widely used quantum-mechanical (QM) approach, where New Physics is parametrized by a set of NSI production and detection parameters, to correctly reproduce the quantum field theory (QFT) result.

The paper has the following structure. In section 2, we discuss how to calculate the differential rate of neutrino oscillation events, starting with a QFT with an arbitrary field content and interaction Lagrangian. The result is encompassed in eq. (2.1), where the rate is expressed by the amplitudes for production and detection of the neutrino. This can be compared with the QM prescription to calculate the same observable, in which the connection with the underlying physics of neutrino interactions is obscure. In section 3, we introduce an EFT Lagrangian that describes interactions between neutrinos, leptons, and quarks. The possible departure of these interactions from the SM predictions is parameterized by a set of Wilson coefficients. The connection between these Wilson coefficients and the rate formula in eq. (2.1) is made transparent by introducing processdependent production and detection coefficients. We also derive the matching between the Wilson coefficients in the EFT, and the familiar NSI parameters in the QM description. The matching is always possible at the linear order in the Wilson coefficients. However, only if the production and detection coefficients satisfy a certain consistency condition, that matching is valid beyond the linear order. In section 4 we calculate the production and detection coefficients for several specific processes of relevance to current neutrino experiments. We cover the cases of neutrinos produced in nuclear beta or pion decays, and detected by inverse beta decay. The formulas for the neutrino oscillation probability are collected in section 5. Section 6 is devoted to the discussion of NSI for neutrinos produced in muon decay. The study of this process requires the introduction of 4-lepton effective operators and introduces new features due to the presence of one neutrino and one antineutrino. Finally, section 7 contains our concluding remarks.

\section{Formalism}

\subsection{QFT description}

Oscillation probability can be rigorously derived in the framework of quantum field theory. Various derivations are available in the literature in the absence of NSI (see e.g. [25-27]). Below we give an expression valid for completely general interactions between neutrinos and matter. Consider neutrinos produced in the process $S \rightarrow X_{\alpha} \nu$ (e.g. beta decay of a nucleus in a reactor, or pion decay), where $X_{\alpha}$ is one or more body final states containing one charged lepton $\ell_{\alpha}=(e, \mu, \tau)$. Neutrinos are detected via the process $\nu T \rightarrow Y_{\beta}$ (e.g. inverse beta decay), where again $Y_{\beta}$ contains a charged lepton $\ell_{\beta}$. The production and detection can be described by QFT amplitudes $\mathcal{M}_{\alpha k}^{P} \equiv \mathcal{M}\left(S \rightarrow X_{\alpha} \nu_{k}\right)$ and $\mathcal{M}_{\beta k}^{D} \equiv \mathcal{M}\left(\nu_{k} T \rightarrow Y_{\beta}\right)$, where the index $k$ labels neutrino mass eigenstates. The information about fundamental parameters, is encoded in $\mathcal{M}_{\alpha k}^{P}$ and $\mathcal{M}_{\beta k}^{D}$, which should be then connected to observables. For source (S) and target (T) states separated by a macroscopic distance $L$, the observable 
is the differential rate of detected events per target particle $R_{\alpha \beta} \equiv \frac{d N_{\alpha \beta}}{N_{T} d t d E_{\nu}}$ given by

$$
R_{\alpha \beta}=\frac{N_{S}}{32 \pi L^{2} m_{S} m_{T} E_{\nu}} \sum_{k, l} e^{-i \frac{L \Delta m_{k l}^{2}}{2 E_{\nu}}} \int d \Pi_{P^{\prime}} \mathcal{M}_{\alpha k}^{P} \overline{\mathcal{M}}_{\alpha l}^{P} \int d \Pi_{D} \mathcal{M}_{\beta k}^{D} \overline{\mathcal{M}}_{\beta l}^{D}
$$

A compact derivation of this formula is presented in appendix A, where we also enumerate its limitations. Above, $\Delta m_{k l}^{2} \equiv m_{k}^{2}-m_{l}^{2}$ is the mass squared difference between neutrino eigenstates. The phase space elements $d \Pi_{P}$ and $d \Pi_{D}$ for the production and detection processes are defined in the standard way: $d \Pi \equiv \frac{d^{3} k_{1}}{(2 \pi)^{3} 2 E_{1}} \cdots \frac{d^{3} k_{n}}{(2 \pi)^{3} 2 E_{n}}(2 \pi)^{4} \delta^{4}\left(\mathcal{P}-\sum k_{i}\right)$, where $\mathcal{P}$ is the total 4 -momentum of the initial state and $k_{i}$ are the 4 -momenta of the final states. The production $d \Pi_{P}$ includes the neutrino phase space $\frac{d^{3} k_{\nu}}{(2 \pi)^{3} 2 E_{\nu}}$ and we define $d \Pi_{P^{\prime}}$ via $d \Pi_{P} \equiv d \Pi_{P^{\prime}} d E_{\nu}$. The amplitudes $\mathcal{M}_{P, D}$ describe the neutrino production and detection processes, which we allow to be arbitrary. The $\int$ sign in eq. (2.1) involves integration and sum/average over all unobserved degrees of freedom, such as angular variables and spins. Finally, complex conjugated amplitudes are denoted with a bar, $N_{S, T}$ are the number of source/target particles, and $m_{S, T}$ are their masses. The derivation of eq. (2.1) assumes that neutrinos are produced by a source at rest and are emitted isotropically; see eq. (A.17) for a more general formula.

The rate in eq. (2.1) displays the famous oscillatory behavior via the $\exp \left(-i \frac{L \Delta m_{k l}^{2}}{2 E_{\nu}}\right)$ factor. In the absence of oscillations, rates would be calculated using the neutrino differential flux $\Phi_{\alpha} \equiv \frac{N_{S}}{4 \pi L^{2}} \frac{d \Gamma_{\alpha}^{P}}{d E_{\nu}}$, and the detection cross section at the target. The source decay rate $\Gamma_{\alpha}^{P}$ (with emission of $\ell_{\alpha}$ and summed over neutrino mass eigenstates) and the detection cross section $\sigma_{\beta}^{D}$ (with emission of $\ell_{\beta}$ and summed over neutrino mass eigenstates) can be calculated by the usual means in QFT. We have

$$
\Phi_{\alpha} \sigma_{\beta}=\frac{N_{S}}{32 \pi L^{2} m_{S} m_{T} E_{\nu}} \int d \Pi_{P^{\prime}} \sum_{k}\left|\mathcal{M}_{\alpha k}^{P}\right|^{2} \int d \Pi_{D} \sum_{l}\left|\mathcal{M}_{\beta l}^{D}\right|^{2} .
$$

One can define the $\nu_{\alpha} \rightarrow \nu_{\beta}$ oscillation probability as the ratio of the rate of detected events in eq. (2.1) to the no-oscillation expression in eq. (2.2), finding

$$
P_{\alpha \beta}=\frac{\sum_{k, l} e^{-i \frac{L \Delta m_{k l}^{2}}{2 E_{\nu}}} \int d \Pi_{P^{\prime}} \mathcal{M}_{\alpha k}^{P} \overline{\mathcal{M}}_{\alpha l}^{P} \int d \Pi_{D} \mathcal{M}_{\beta k}^{D} \overline{\mathcal{M}}_{\beta l}^{D}}{\int d \Pi_{P^{\prime}} \sum_{k}\left|\mathcal{M}_{\alpha k}^{P}\right|^{2} \int d \Pi_{D} \sum_{l}\left|\mathcal{M}_{\beta l}^{D}\right|^{2}}
$$

This formula appears in ref. [28] in a slightly different form without explicit phase space integration. Oscillation probability is an intuitive and widely employed concept, however strictly speaking $P_{\alpha \beta}$ is not an observable. For this reason in this paper we work mainly with the rate in eq. (2.1). Nonetheless, the oscillation probability is also discussed in section 5 .

\subsection{QM-NSI description}

Neutrino oscillations are often described in a simple quantum mechanical setting. One defines flavor states as linear combinations of mass eigenstates: $\left|\nu_{\alpha}\right\rangle=\sum_{k} U_{\alpha k}\left|\nu_{k}\right\rangle$, where $U$ is the unitary PMNS mixing matrix. In this language, NSI effects are encoded in 
parameters $\epsilon_{\alpha \beta}^{s, d}[19,29,30]$, which are defined by the mismatch between pure flavor states and neutrino states produced at the source and detected at the target, namely [9]:

$$
\left|\nu_{\alpha}^{s}\right\rangle=\frac{\left(1+\epsilon^{s}\right)_{\alpha \gamma}}{N_{\alpha}^{s}}\left|\nu_{\gamma}\right\rangle, \quad\left\langle\nu_{\beta}^{d}\right|=\left\langle\nu_{\gamma}\right| \frac{\left(1+\epsilon^{d}\right)_{\gamma \beta}}{N_{\beta}^{d}},
$$

with the normalization $N_{\alpha}^{s}=\sqrt{\left[\left(1+\epsilon^{s}\right)\left(1+\epsilon^{s \dagger}\right)\right]_{\alpha \alpha}}, N_{\beta}^{d}=\sqrt{\left[\left(1+\epsilon^{d \dagger}\right)\left(1+\epsilon^{d}\right)\right]_{\beta \beta}}$. The probability of $\left|\nu_{\alpha}^{s}\right\rangle$ oscillating into $\left|\nu_{\beta}^{d}\right\rangle$ is given by $P_{\alpha \beta}^{Q M}=\left|\left\langle\nu_{\beta}^{d}\left|e^{-i H L}\right| \nu_{\alpha}^{s}\right\rangle\right|^{2}$, where the Hamiltonian is $H_{\beta \alpha}=\sum_{k} U_{\beta k} m_{k}^{2} U_{\alpha k}^{*} /\left(2 E_{\nu}\right)$ in the absence of matter effects in propagation. In this approach, which we refer to as QM-NSI formalism, the event rate is given by [6]

$$
\begin{aligned}
R_{\alpha \beta}^{\mathrm{QM}} & =\Phi_{\alpha}^{\mathrm{SM}} \sigma_{\beta}^{\mathrm{SM}} P_{\alpha \beta}^{\mathrm{QM}}\left(N_{\alpha}^{s} N_{\beta}^{d}\right)^{2} \\
& =\Phi_{\alpha}^{\mathrm{SM}} \sigma_{\beta}^{\mathrm{SM}} \sum_{k, l} e^{-i \frac{L \Delta m_{k l}^{2}}{2 E_{\nu}}}\left[x_{s}\right]_{\alpha k}\left[x_{s}\right]_{\alpha l}^{*}\left[x_{d}\right]_{\beta k}\left[x_{d}\right]_{\beta l}^{*},
\end{aligned}
$$

where $x_{s} \equiv\left(1+\epsilon^{s}\right) U^{*}$ and $x_{d} \equiv\left(1+\epsilon^{d}\right)^{T} U$. For antineutrinos eq. (2.5) holds with $U \rightarrow U^{*}$. Above, $\Phi_{\alpha}^{\mathrm{SM}}$ and $\sigma_{\beta}^{\mathrm{SM}}$ are the incident flux and detection cross section calculated in the absence of NSI. Normalization factors $N_{\alpha}^{s} N_{\beta}^{d}$ cancel in the observable rate and thus one could have omitted them altogether [6]; their only role is to ensure that $P_{\alpha \beta} \leq 1$, that is it can be interpreted as a probability.

Results from oscillation experiments are often presented or recast as constraints on the NSI parameters $\epsilon_{\alpha \beta}^{s, d}$. However, the utility of the latter hinges on whether they can be unambiguously connected to more fundamental parameters in a Lagrangian. Only after such matching the coefficients $\epsilon_{\alpha \beta}^{s, d}$ determined in different experimental settings can be meaningfully compared and combined. In the following we discuss this issue, and illustrate it with physically relevant examples. We will define the conditions under which the NSI parameters can indeed provide an adequate description of NP effects in neutrino oscillation. Conversely, we will show examples where this is not the case.

\section{Matching QFT and QM-NSI results}

One could try to match the QM-NSI and QFT language starting from the definition in eq. (2.4). This however would be problematic, as such concepts as neutrino flavor states or production and detection states are murky in a QFT framework when general chargedcurrent neutrino interactions with matter are allowed. Therefore, we will follow a pragmatic approach and match the observable rates predicted by the QFT (eq. (2.1)) and QM-NSI frameworks (eq. (2.5)). This comparison will allow us to determine the map between the NSI $\epsilon^{s, d}$ and the Lagrangian parameters, or else conclude the map does not exist.

Thus, in this paper we focus on NP in charged-current interactions between neutrino and matter. The theory framework we consider is the EFT of the SM degrees of freedom at the energy scale $\mu \approx 2 \mathrm{GeV}$, in which lepton-number conserving NP modifies the effective 4 -fermion interactions between leptons and quarks. Extensions to other theories and interactions are straightforward using our approach. For example, introducing right-handed 
neutrinos interacting with matter or additional sterile neutrinos mixing with the active ones, would not bring any qualitative change to the formalism.

At leading order in our EFT neutrino interactions with matter can be parametrized by the Lagrangian (see e.g. ref. [31])

$$
\begin{aligned}
\mathcal{L} \supset-\frac{2 V_{u d}}{v^{2}}\{ & {\left[\mathbf{1}+\epsilon_{L}\right]_{\alpha \beta}\left(\bar{u} \gamma^{\mu} P_{L} d\right)\left(\bar{\ell}_{\alpha} \gamma_{\mu} P_{L} \nu_{\beta}\right) } \\
& +\left[\epsilon_{R}\right]_{\alpha \beta}\left(\bar{u} \gamma^{\mu} P_{R} d\right)\left(\bar{\ell}_{\alpha} \gamma_{\mu} P_{L} \nu_{\beta}\right) \\
& +\frac{1}{2}\left[\epsilon_{S}\right]_{\alpha \beta}(\bar{u} d)\left(\bar{\ell}_{\alpha} P_{L} \nu_{\beta}\right)-\frac{1}{2}\left[\epsilon_{P}\right]_{\alpha \beta}\left(\bar{u} \gamma_{5} d\right)\left(\bar{\ell}_{\alpha} P_{L} \nu_{\beta}\right) \\
& \left.+\frac{1}{4}\left[\epsilon_{T}\right]_{\alpha \beta}\left(\bar{u} \sigma^{\mu \nu} P_{L} d\right)\left(\bar{\ell}_{\alpha} \sigma_{\mu \nu} P_{L} \nu_{\beta}\right)+\text { h.c. }\right\},
\end{aligned}
$$

where $v \equiv\left(\sqrt{2} G_{F}\right)^{-1 / 2} \approx 246 \mathrm{GeV}, V_{u d}$ is a CKM matrix element, $\sigma^{\mu \nu}=i\left[\gamma^{\mu}, \gamma^{\nu}\right] / 2$, and $P_{L, R}=\left(1 \mp \gamma_{5}\right) / 2$. The quarks $u, d$, and charged leptons $\ell_{\alpha}$ are in the basis where their kinetic and mass terms are diagonal. For neutrino fields kinetic terms are diagonal but mass terms are not, thus $\nu_{\alpha}$ are connected to mass eigenstates by the unitary PMNS rotation $\nu_{\alpha}=\sum_{k} U_{\alpha k} \nu_{k}$. In this EFT the effects of NP are parametrized by the Wilson coefficients $\left[\epsilon_{X}\right]_{\alpha \beta}$, which encode new interactions between quarks and leptons mediated by BSM particles heavier than $\sim 2 \mathrm{GeV}$. For example, non-zero $\epsilon_{R}$ can arise in left-right symmetric models due to the $W_{R}$ boson coupling to right-handed quarks and mixing with the SM $W^{ \pm}$, while non-zero $\epsilon_{S, P, T}$ are generally predicted in leptoquarks models. More generally, $\epsilon_{X}$ can be connected to parameters of the weak-scale EFT, known as the SMEFT [31-33]. The constraints obtained from neutrino oscillations may have an impact on these broad classes of models.

We remark that, in the EFT below the weak scale where charged and neutral leptons are not collected into doublets, nothing distinguishes the basis of $\nu_{\alpha}$ in eq. (3.1) as soon as $\left[\epsilon_{L}\right]_{\alpha \beta} \neq 0$. Unitary rotations $\nu_{\alpha} \rightarrow V \nu_{\alpha}$ transform the Lagrangian into an equivalent basis with NP parameters rotated as $\delta_{X, L}+\epsilon_{X} \rightarrow\left(\delta_{X, L}+\epsilon_{X}\right) V$, and the neutrino mass matrix rotated by $M_{\nu} \rightarrow V^{T} M_{\nu} V$. Physics of course cannot depend on which basis we work with. We will see that observable rates will be invariant under such rotations of $\epsilon_{X}$ accompanied by rotating the PMNS matrix $U \rightarrow V^{\dagger} U$.

\subsection{SM interactions}

To warm up, let us first calculate the event rate in the limit of the SM interactions, which corresponds to setting all $\epsilon_{X}=0$. In this case, which was studied in ref. [25], the amplitudes can be decomposed as:

$$
\mathcal{M}_{\alpha k}^{P}=U_{\alpha k}^{*} A_{L}^{P}, \quad \mathcal{M}_{\alpha k}^{D}=U_{\alpha k} A_{L}^{D}
$$

The functions $A_{L}^{P, D}$ are independent of the neutrino mass index $k$ up to negligible corrections, whereas they do depend on the charged-lepton flavor index $\alpha$ (which we omit to ease the notation). They also depend on the kinematic and spin variables in the production and detection processes, and they appear in the observables integrated/averaged over by 
$\int d \Pi_{P^{\prime}, D}$. All in all the rate in eq. (2.1) can be written as

$$
R_{\alpha \beta}^{\mathrm{SM}}=\Phi_{\alpha}^{\mathrm{SM}} \sigma_{\beta}^{\mathrm{SM}} \sum_{k, l} e^{-i \frac{L \Delta m_{k l}^{2}}{2 E_{\nu}}} U_{\alpha k}^{*} U_{\alpha l} U_{\beta k} U_{\beta l}^{*},
$$

where the SM flux and cross-section are given by

$$
\Phi_{\alpha}^{\mathrm{SM}}=\frac{N_{S} \int d \Pi_{P^{\prime}}\left|A_{L}^{P}\right|^{2}}{8 m_{S} \pi L^{2}}, \quad \sigma_{\beta}^{\mathrm{SM}}=\frac{\int d \Pi_{D}\left|A_{L}^{D}\right|^{2}}{4 E_{\nu} m_{T}} .
$$

Exactly the same result is obtained from eq. (2.5) in the limit $\epsilon_{\alpha \beta}^{s, d}=0$.

\section{$3.2 \quad V-A$ interactions}

A less trivial example is when NP enters only via $V$ - $A$ interactions: $\left[\epsilon_{L}\right]_{\alpha \beta} \neq 0[5,6,34,35]$. In this case the detection/production amplitudes decompose as

$$
\mathcal{M}_{\alpha k}^{P}=\left[\left(1+\epsilon_{L}\right) U\right]_{\alpha k}^{*} A_{L}^{P}, \quad \mathcal{M}_{\alpha k}^{D}=\left[\left(1+\epsilon_{L}\right) U\right]_{\alpha k} A_{L}^{D} .
$$

We obtain

$$
R_{\alpha \beta}^{V-A}=\Phi_{\alpha}^{\mathrm{SM}} \sigma_{\beta}^{\mathrm{SM}} \sum_{k, l} e^{-i \frac{L \Delta m_{k l}^{2}}{2 E_{\nu}}}\left[x_{L}\right]_{\alpha k}^{*}\left[x_{L}\right]_{\alpha l}\left[x_{L}\right]_{\beta k}\left[x_{L}\right]_{\beta l}^{*},
$$

where $x_{L} \equiv\left(1+\epsilon_{L}\right) U$. In fact, the quantity $x_{L}$ is equivalent to a "non-unitary mixing matrix", an approach that has been studied in the neutrino literature [6, 35-37]. The same result is obtained in the QM-NSI approach from eq. (2.5) when NSI parameters are mapped to the Lagrangian parameters as [35]:

$$
\mathbf{V}-\mathbf{A}: \quad \epsilon_{\alpha \beta}^{s}=\left[\epsilon_{L}\right]_{\alpha \beta}^{*}, \quad \epsilon_{\beta \alpha}^{d}=\left[\epsilon_{L}\right]_{\alpha \beta} .
$$

In the $V$ - $A$ case the map between NSI and Lagrangian parameters is well-defined, unambiguous, and simple.

\subsection{General case}

For general NP interactions in eq. (3.1), the production and detection amplitudes can be decomposed as

$$
\begin{aligned}
& \mathcal{M}_{\alpha k}^{P}=U_{\alpha k}^{*} A_{L}^{P}+\sum_{X}\left[\epsilon_{X} U\right]_{\alpha k}^{*} A_{X}^{P}, \\
& \mathcal{M}_{\beta k}^{D}=U_{\beta k} A_{L}^{D}+\sum_{X}\left[\epsilon_{X} U\right]_{\beta k} A_{X}^{D} .
\end{aligned}
$$

The sum above goes over all types of interactions in eq. (3.1): $X=L, R, S, P, T$. We stress that $A_{X}^{P, D}$ will typically have completely different dependence on kinematic and spin variables for different $X$. Plugging this decomposition into eq. (2.1) we obtain

$$
\begin{aligned}
R_{\alpha \beta}= & \Phi_{\alpha}^{\mathrm{SM}} \sigma_{\beta}^{\mathrm{SM}} \sum_{k, l} e^{-i \frac{L \Delta m_{k l}^{2}}{2 E_{\nu}}} \\
& \times\left[U_{\alpha k}^{*} U_{\alpha l}+p_{X L}\left(\epsilon_{X} U\right)_{\alpha k}^{*} U_{\alpha l}+p_{X L}^{*} U_{\alpha k}^{*}\left(\epsilon_{X} U\right)_{\alpha l}+p_{X Y}\left(\epsilon_{X} U\right)_{\alpha k}^{*}\left(\epsilon_{Y} U\right)_{\alpha l}\right] \\
& \times\left[U_{\beta k} U_{\beta l}^{*}+d_{X L}\left(\epsilon_{X} U\right)_{\beta k} U_{\beta l}^{*}+d_{X L}^{*} U_{\beta k}\left(\epsilon_{X} U\right)_{\beta l}^{*}+d_{X Y}\left(\epsilon_{X} U\right)_{\beta k}\left(\epsilon_{Y} U\right)_{\beta l}^{*}\right],
\end{aligned}
$$


where we define the production and detection coefficients

$$
p_{X Y} \equiv \frac{\int d \Pi_{P^{\prime}} A_{X}^{P} \bar{A}_{Y}^{P}}{\int d \Pi_{P^{\prime}}\left|A_{L}^{P}\right|^{2}}, \quad d_{X Y} \equiv \frac{\int d \Pi_{D} A_{X}^{D} \bar{A}_{Y}^{D}}{\int d \Pi_{D}\left|A_{L}^{D}\right|^{2}} .
$$

We show in section 4 the expressions of the above coefficients for different processes. For antineutrinos eq. (3.9) holds with $U \leftrightarrow U^{*}$ and $\epsilon_{X} \leftrightarrow \epsilon_{X}^{*}$. The formulas for the neutrino oscillation probability are collected in section 5 .

At linear level in $\epsilon$ the QFT expression in eq. (3.9) matches the QM-NSI one in eq. (2.5) provided NSI parameters are expressed by the EFT parameters as

$$
\epsilon_{\alpha \beta}^{s}=\sum_{X} p_{X L}\left[\epsilon_{X}\right]_{\alpha \beta}^{*}, \quad \epsilon_{\beta \alpha}^{d}=\sum_{X} d_{X L}\left[\epsilon_{X}\right]_{\alpha \beta} .
$$

Therefore, the QM-NSI formalism can approximate the correct oscillation probability obtained from the general EFT as long as the deviation from the SM, encoded in the coefficients $\left[\epsilon_{X}\right]_{\alpha \beta}$, is sufficiently small. If non-SM-like interactions are involved (that is with a different Lorentz structure than $V$-A), the NSI parameters obtained via the matching in eq. (3.11) may be a function of the neutrino energy and they do not satisfy anymore the relation $\epsilon^{s}=\epsilon^{d \dagger}$ valid for the $V$ - $A$ case.

Beyond the linear approximation the QM-NSI formalism fails in general because no matching can be found to connect with the QFT result. The consistency condition for the matching in eq. (3.11) to be valid to all orders in $\epsilon$ is

$$
p_{X L} p_{Y L}^{*}=p_{X Y}, \quad d_{X L} d_{Y L}^{*}=d_{X Y}
$$

for all $X$ and $Y$ for which $\epsilon_{X, Y}$ are non-zero. Eq. (3.12) is trivially satisfied if the only NP deformations are of the $V$ - $A$ type, that is if only $\epsilon_{L}$ is non-zero, in agreement with our previous discussion. However, for non-SM-like deformations eq. (3.12) is typically not satisfied, because then $A_{X}^{P, D}$ may have different dependence on kinematic variables than $A_{L}^{P, D}$.

In the next section we look at specific processes and give concrete examples where eq. (3.12) are not satisfied. We also show cases where the conditions do hold.

\section{Application to specific processes}

The matching between the NSI parameters $\epsilon^{s}$ and $\epsilon^{d}$ to the EFT Wilson Coefficients $\epsilon_{X}$ depends on the specific processes in which neutrinos are produced or detected, as shown in eq. (3.11). The process dependence is encoded in the production and detection coefficients $p_{X Y}$ and $d_{X Y}$ defined in eq. (3.10). With the production and detection coefficients at hand, we can verify whether the consistency condition in eq. (3.12) is satisfied. If it is not, the matching is only valid at the linear order in $\epsilon_{X}$, whereas at higher orders it fails. In the latter case, the QM-NSI approach does not reproduce the correct dependence on EFT parameters beyond the linear order in $\epsilon_{X}$. Here we list the production and detection coefficients for inverse beta decay, nuclear decay, and pion decay, and discuss the validity of the matching in each case. The discussion of muon decay is left for section 6 . Table 1 


\begin{tabular}{|c|c|}
\hline Neutrino process & NSI matching with EFT \\
\hline$\nu_{e}$ produced in beta decay & $\epsilon_{e \beta}^{s}=\left[\epsilon_{L}-\epsilon_{R}-\frac{g_{T}}{g_{A}} \frac{m_{e}}{f_{T}\left(E_{\nu}\right)} \epsilon_{T}\right]_{e \beta}^{*}$ \\
\hline$\nu_{e}$ detected in inverse beta decay & $\epsilon_{\beta e}^{d}=\left[\epsilon_{L}+\frac{1-3 g_{A}^{2}}{1+3 g_{A}^{2}} \epsilon_{R}-\frac{m_{e}}{E_{\nu}-\Delta}\left(\frac{g_{S}}{1+3 g_{A}^{2}} \epsilon_{S}-\frac{3 g_{A} g_{T}}{1+3 g_{A}^{2}} \epsilon_{T}\right)\right]_{e \beta}$ \\
\hline$\nu_{\mu}$ produced in pion decay & $\epsilon_{\mu \beta}^{s}=\left[\epsilon_{L}-\epsilon_{R}-\frac{m_{\pi}^{2}}{m_{\mu}\left(m_{u}+m_{d}\right)} \epsilon_{P}\right]_{\mu \beta}^{*}$ \\
\hline$\nu_{\mu}$ and $\bar{\nu}_{e}$ produced in muon decay & $\epsilon_{\mu \beta}^{s}=\left[\rho_{L}-\frac{3 m_{e}}{3 m_{\mu}-4 E_{\nu}} \rho_{R}\right]_{e e \beta \mu}$ \\
& $\epsilon_{e \beta}^{s}=\left[\rho_{L}-\frac{m_{e}}{2 m_{\mu}-4 E_{\bar{\nu}}} \rho_{R}\right]_{e \beta \mu \mu}$ \\
\hline
\end{tabular}

Table 1. Summary of the matching between NSI parameters and EFT Wilson coefficients. See section 4 and section 6 for further details about the validity of the QM-NSI approach in each case and for the definition of the Wilson coefficients $\rho$ relevant for muon decay. For antineutrinos matching is the same up to complex conjugation.

summarizes the linear matching between the NSI parameters and EFT Wilson coefficients for these processes.

Neutrino detection through non-elastic processes (quasi-elastic, deep-inelastic, or resonances) are more complicated, and even in the SM it is often challenging to provide accurate predictions, mainly due to nontrivial hadronic/nuclear physics involved. For this reason, we leave these processes for future work.

A common detection process of low-energy neutrinos is the inverse beta decay, $\nu p \rightarrow$ $n e$. For this case we find the following detection coefficients:

$$
\begin{array}{ll}
d_{L L}=1, \quad d_{R L}=\frac{1-3 g_{A}^{2}}{1+3 g_{A}^{2}}, & d_{S L}=d_{S R}=-\frac{g_{S}}{1+3 g_{A}^{2}} \frac{m_{e}}{E_{\nu}-\Delta}, \\
d_{T L}=-d_{T R}=\frac{3 g_{A} g_{T}}{1+3 g_{A}^{2}} \frac{m_{e}}{E_{\nu}-\Delta}, & \\
d_{R R}=1, \quad d_{S S}=\frac{g_{S}^{2}}{1+3 g_{A}^{2}}, & d_{T T}=\frac{3 g_{T}^{2}}{1+3 g_{A}^{2}},
\end{array}
$$

where $g_{A}=1.251(33), g_{S}=1.02(10), g_{T}=0.987(55)$ are the axial, scalar and tensor nucleon charges [38-41], $\Delta=m_{n}-m_{p} \sim 1.3 \mathrm{MeV}$ is the neutron-proton mass difference and $m_{e}$ is the electron mass. We note that the usual chiral factor $\sim m_{\ell} / E_{\nu}$ associated to (pseudo-)scalar and tensor interactions [7, 42] is of order one in this case.

The NSI detection parameters can thus be related to the EFT parameters as

$$
\begin{aligned}
\epsilon_{\beta e}^{d} & =\sum_{X} d_{X L}\left[\epsilon_{X}\right]_{e \beta} \\
& =\left[\epsilon_{L}\right]_{e \beta}+\frac{1-3 g_{A}^{2}}{1+3 g_{A}^{2}}\left[\epsilon_{R}\right]_{e \beta}-\frac{m_{e}}{E_{\nu}-\Delta}\left(\frac{g_{S}}{1+3 g_{A}^{2}}\left[\epsilon_{S}\right]_{e \beta}-\frac{3 g_{A} g_{T}}{1+3 g_{A}^{2}}\left[\epsilon_{T}\right]_{e \beta}\right) .
\end{aligned}
$$


The consistency condition in eq. (3.12) is satisfied only for the $V$ - $A$ case, and fails if other NSIs are present. For example, consider NP of the $V+A$ type affecting the process, that is $\left[\epsilon_{R}\right]_{e \beta} \neq 0$ for some $\beta$. Since $\left|d_{R L}\right|^{2} \neq d_{R R}$, we conclude that the effect of $V+A$ interactions in neutrino experiments that involve inverse beta decay cannot be described by the NSI parameters $\epsilon^{s, d}$ beyond the linear level.

In the presence of scalar and tensor interactions we have again $\left|d_{S L}\right|^{2} \neq d_{S S},\left|d_{T L}\right|^{2} \neq$ $d_{T T}$. Moreover, in these two cases the left-hand sides depend on the neutrino energy, while the right-hand sides do not.

Reactor electron antineutrinos $\bar{\nu}_{e}$ are produced via beta decays of nuclear fission products. To calculate the corresponding amplitudes we assume that only the Gamow-Teller type decays are important (see ref. [32] for further details). With this assumption the non-zero coefficients are

$$
\begin{array}{ll}
p_{L L}=-p_{R L}=1, & p_{T L}=-p_{T R}=-\frac{g_{T}}{g_{A}} \frac{m_{e}}{f_{T}\left(E_{\nu}\right)}, \\
p_{R R}=1, & p_{T T}=\frac{g_{T}^{2}}{g_{A}^{2}}
\end{array}
$$

which gives the following matching with NSI parameters

$$
\epsilon_{e \beta}^{s}=\sum_{X} p_{X L}\left[\epsilon_{X}\right]_{e \beta}^{*}=\left[\epsilon_{L}\right]_{e \beta}^{*}-\left[\epsilon_{R}\right]_{e \beta}^{*}-\frac{g_{T}}{g_{A}} \frac{m_{e}}{f_{T}\left(E_{\nu}\right)}\left[\epsilon_{T}\right]_{e \beta}^{*} .
$$

Here $f_{T}\left(E_{\nu}\right)$ is a function that depends on the nuclear decays taking place in the reactor, which was calculated using certain approximations in ref. [32]. We see that the relation in eq. (3.12) is not satisfied for the tensor case: $\frac{m_{e}^{2}}{f_{T}^{2}\left(E_{\nu}\right)} \neq 1$, which implies that reactor antineutrino production cannot be fully described by the QM-NSI formalism in the presence of tensor interactions. Moreover, the energy dependence at the linear level (entering via $p_{T L}$ ) is not there at the quadratic level (because $p_{T T}$ is a constant), which will be missed if we use eq. (4.4) in eq. (2.5). For the left- and right-handed interactions the matching is valid at all orders.

We see in this last example that one should not jump into the conclusion that the QMNSI formalism always fails for non-SM-like interactions. For instance, this is not the case if $A_{X}^{P, D}=c_{X}^{P, D} A_{L}^{P, D}$, where $c_{X}^{P, D}$ is a constant independent of the kinematic variables to be integrated over. The latter happens e.g. in the 2-body decay of a spin-zero particle. This includes of course the phenomenologically relevant case of neutrino production through pion decays. Thanks to the pseudoscalar nature of the pion, the only non-zero hadronic matrix elements for this decay are $\left\langle 0\left|\bar{u} \gamma^{\mu} \gamma_{5} d\right| \pi^{+}\right\rangle$and $\left\langle 0\left|\bar{u} \gamma_{5} d\right| \pi^{+}\right\rangle$. As a result the production process is sensitive only to axial $\left(\epsilon_{L^{-}} \epsilon_{R}\right)$ and pseudo-scalar $\left(\epsilon_{P}\right)$ interactions.

For pions at rest, the non-zero production coefficients are

$$
\begin{array}{ll}
p_{L L}=-p_{R L}=1, & p_{P L}=-p_{P R}=-\frac{m_{\pi}^{2}}{m_{\mu}\left(m_{u}+m_{d}\right)}, \\
p_{R R}=1, & p_{P P}=\frac{m_{\pi}^{4}}{m_{\mu}^{2}\left(m_{u}+m_{d}\right)^{2}} .
\end{array}
$$


The NSI production parameters can thus be related to the EFT parameters as

$$
\epsilon_{\mu \beta}^{s}=\sum_{X} p_{X L}\left[\epsilon_{X}\right]_{\mu \beta}^{*}=\left[\epsilon_{L}\right]_{\mu \beta}^{*}-\left[\epsilon_{R}\right]_{\mu \beta}^{*}-\frac{m_{\pi}^{2}}{m_{\mu}\left(m_{u}+m_{d}\right)}\left[\epsilon_{P}\right]_{\mu \beta}^{*} .
$$

We see that the consistency condition in eq. (3.12) is satisfied for all the interactions involved in pion decay. Therefore, neutrino production via pion decays can be described by the QM-NSI formalism to all orders (with the above matching), even in the presence of non-SM-like interactions.

\section{Oscillation probability}

So far in this paper the basic quantity we have worked with was the event rate $R_{\alpha \beta}$ in eq. (2.1), which is an observable in neutrino experiments. This quantity can be decomposed into the product of the oscillation probability (eq. (2.3)) and the no-oscillation result (eq. (2.2)):

$$
R_{\alpha \beta}=P_{\alpha \beta} \times \Phi_{\alpha} \sigma_{\beta}
$$

In this section we the expressions for the oscillation probability $P_{\alpha \beta}$ in both QM-NSI and QFT frameworks, and we also discuss some relevant features. From the general QFT viewpoint, the decomposition in eq. (5.1) may seem artificial, as the rate in eq. (2.1) is directly observable in neutrino experiments. Nonetheless, there are advantages of defining the oscillation probability that go beyond its obvious intuitive qualities. First, for the sake of calculating ratios of measurements at different distances $L$ for a fixed $E_{\nu}$, the ratio of probabilities is the same as the ratio of rates. Second, in many familiar scenarios the physics contributing to the oscillation probability in eq. (2.3) and no-oscillation piece in eq. (2.2) is distinct. In the SM, electroweak and hadronic parameters contribute to the flux and cross-section, while neutrino masses and mixing angles contribute to the oscillation probability. In the EFT scenario (3.1) at the linear level only flavor off-diagonal $\epsilon_{X}$ affect the oscillation probability, whereas flavor diagonal $\epsilon_{X}$ affect the flux/cross section [32]. It is important to note however that such a separation does not hold beyond the linear level.

In the QM-NSI approach the oscillation probability is given by

$$
P_{\alpha \beta}^{\mathrm{QM}}=\left(N_{\alpha}^{s} N_{\beta}^{d}\right)^{-2} \sum_{k, l} e^{-i \frac{L \Delta m_{k l}^{2}}{2 E_{\nu}}}\left[x_{s}\right]_{\alpha k}\left[x_{s}\right]_{\alpha l}^{*}\left[x_{d}\right]_{\beta k}\left[x_{d}\right]_{\beta l}^{*} .
$$

As discussed in section 2, we have $x_{s} \equiv\left(1+\epsilon^{s}\right) U^{*}$ and $x_{d} \equiv\left(1+\epsilon^{d}\right)^{T} U$, and the normalization factors are

$$
\left(N_{\alpha}^{s} N_{\beta}^{d}\right)^{2}=\left[\left(1+\epsilon^{s}\right)\left(1+\epsilon^{s \dagger}\right)\right]_{\alpha \alpha}\left[\left(1+\epsilon^{d \dagger}\right)\left(1+\epsilon^{d}\right)\right]_{\beta \beta} .
$$


In the QFT approach the oscillation probability depends on the parameters of the EFT Lagrangian in eq. (3.1) as

$$
\begin{aligned}
P_{\alpha \beta}^{\mathrm{QFT}}= & N_{\alpha \beta}^{-1} \sum_{k, l} e^{-i \frac{L \Delta m_{k l}^{2}}{2 E_{\nu}}} \\
& \times\left[U_{\alpha k}^{*} U_{\alpha l}+\sum_{X} p_{X L}\left(\epsilon_{X} U\right)_{\alpha k}^{*} U_{\alpha l}+\sum_{X} p_{X L}^{*} U_{\alpha k}^{*}\left(\epsilon_{X} U\right)_{\alpha l}+\sum_{X, Y} p_{X Y}\left(\epsilon_{X} U\right)_{\alpha k}^{*}\left(\epsilon_{Y} U\right)_{\alpha l}\right] \\
& \times\left[U_{\beta k} U_{\beta l}^{*}+\sum_{X} d_{X L}\left(\epsilon_{X} U\right)_{\beta k} U_{\beta l}^{*}+\sum_{X} d_{X L}^{*} U_{\beta k}\left(\epsilon_{X} U\right)_{\beta l}^{*}+\sum_{X, Y} d_{X Y}\left(\epsilon_{X} U\right)_{\beta k}\left(\epsilon_{Y} U\right)_{\beta l}^{*}\right]
\end{aligned}
$$

where the coefficients $p_{X Y}$ and $d_{X Y}$ are defined in eq. (3.10) and the normalization factor is

$$
\begin{aligned}
N_{\alpha \beta}= & {\left[\mathbf{1}+\sum_{X} p_{X L} \epsilon_{X}^{*}+\sum_{X} p_{X L}^{*} \epsilon_{X}+\sum_{X, Y} p_{X Y} \epsilon_{Y} \epsilon_{X}^{\dagger}\right]_{\alpha \alpha} } \\
& \times\left[\mathbf{1}+\sum_{X} d_{X L} \epsilon_{X}+\sum_{X} d_{X L}^{*} \epsilon_{X}^{*}+\sum_{X, Y} d_{X Y} \epsilon_{X} \epsilon_{Y}^{\dagger}\right]_{\beta \beta} .
\end{aligned}
$$

The QM-NSI and QFT probabilities can be matched as in eq. (3.11) only when the conditions $p_{X Y}=p_{X L} p_{Y L}^{*}$ and $d_{X Y}=d_{X L} d_{Y L}^{*}$ are satisfied for each $X, Y$ for which $\epsilon_{X, Y}$ are non-zero. In the case of $V$-A interactions we have $p_{L L}=d_{L L}=1$ and the consistency conditions are automatically satisfied. The SM limit corresponds to $\epsilon_{X}=0$ in the EFT, or $\epsilon^{s}=\epsilon^{d}=0$ in the QM-NSI approach, in which case we recover the familiar expression

$$
P_{\alpha \beta}^{\mathrm{SM}}=\sum_{k, l} e^{-i \frac{L \Delta m_{k l}^{2}}{2 E_{\nu}}} U_{\alpha k}^{*} U_{\alpha l} U_{\beta k} U_{\beta l}^{*} .
$$

It is well-known that NP can affect the oscillation probability at zero distance, i.e., $\mathrm{P}_{\alpha \beta}(L=0) \neq \delta_{\alpha \beta}[43]$. We find that:

- There are no such "zero-distance effects" at linear order in NP. Let us note that in the $\alpha=\beta$ case the rate itself is affected by linear effects in $\left[\epsilon_{X}\right]_{\alpha \alpha}$, but they come from NP modifying the neutrino flux and detection cross-section in eq. (2.2).

- At quadratic order, zero-distance effects do appear in general.

- Zero-distance effects vanish at all orders in the $\alpha=\beta$ case with $V$ - $A$ interactions, i.e. $\mathrm{P}_{\alpha \alpha}^{V-A}(L=0)=1$.

Our results are therefore relevant for the study of zero-distance effects since they are quadratic and, in the $\alpha=\beta$ case, necessarily non-SM-like. 


\section{Muon decay}

Finally, we consider neutrino production from muon decay (at rest). ${ }^{1}$ For this purpose we need to extend our EFT so as to describe the low-energy neutrino interactions with leptons. At the leading order they can be parametrized as

$$
\mathcal{L} \supset-\frac{2}{v^{2}}\left[\left(\delta_{\alpha a} \delta_{\beta b}+\left[\rho_{L}\right]_{a \alpha \beta b}\right)\left(\bar{\ell}_{a} \gamma^{\rho} P_{L} \nu_{\alpha}\right)\left(\bar{\nu}_{\beta} \gamma_{\rho} P_{L} \ell_{b}\right)-2\left[\rho_{R}\right]_{a \alpha \beta b}\left(\bar{\ell}_{a} P_{L} \nu_{\alpha}\right)\left(\bar{\nu}_{\beta} P_{R} \ell_{b}\right)\right],
$$

where $\left[\rho_{X}\right]_{a \alpha \beta b}^{*}=\left[\rho_{X}\right]_{b \beta \alpha a}$ for the Lagrangian to be Hermitian. A complication in muon decay is that both a neutrino and an antineutrino are produced in the same process. In the following we present the rate $R$ of detecting a neutrino of flavor $\beta$ summed over all antineutrino eigenstates, and the rate $\bar{R}$ of detecting an antineutrino of flavor $\beta$ summed over all neutrino eigenstates. For simplicity, here we neglect new physics in detection. ${ }^{2}$ We find

$$
\begin{aligned}
R_{\mu \beta}= & \Phi^{\mathrm{SM}} \sigma_{\beta}^{\mathrm{SM}} \sum_{k, l} e^{-i \frac{L \Delta m_{k l}^{2}}{2 E_{\nu}}} U_{\beta k} U_{\beta l}^{*} \\
& \times\left[U_{\mu k}^{*} U_{\mu l}+p_{X L}\left[\rho_{X} U^{*}\right]_{e e \mu k} U_{\mu l}+p_{L X} U_{\mu k}^{*}\left[\rho_{X} U^{*}\right]_{e e \mu l}^{*}+\sum_{\gamma} p_{X Y}\left[\rho_{X} U^{*}\right]_{e \gamma \mu k}\left[\rho_{Y} U^{*}\right]_{e \gamma \mu l}^{*}\right], \\
\bar{R}_{e \beta}= & \bar{\Phi}^{\mathrm{SM}} \bar{\sigma}_{\beta}^{\mathrm{SM}} \sum_{k, l} e^{-i \frac{L \Delta m_{k l}^{2}}{2 E_{\nu}}} U_{\beta k}^{*} U_{\beta l} \\
& \times\left[U_{e k} U_{e l}^{*}+\bar{p}_{X L}\left[\rho_{X} U\right]_{e \mu \mu k} U_{e l}^{*}+\bar{p}_{L X} U_{e k}\left[\rho_{X} U\right]_{e \mu \mu l}^{*}+\sum_{\gamma} \bar{p}_{X Y}\left[\rho_{X} U\right]_{e \gamma \mu k}\left[\rho_{Y} U\right]_{e \gamma \mu l}^{*}\right],
\end{aligned}
$$

where we have defined the matrix contractions $\left[\rho_{X} U^{*}\right]_{a \alpha b k} \equiv \sum_{\beta}\left[\rho_{X}\right]_{a \alpha \beta b} U_{\beta k}^{*}$ and $\left[\rho_{X} U\right]_{a \gamma b k} \equiv \sum_{\alpha}\left[\rho_{X}\right]_{a \alpha \gamma b} U_{\alpha k}$. The SM cross sections for detecting a neutrino and an antineutrino of flavor $\beta$ are denoted as $\sigma_{\beta}^{\mathrm{SM}}$ and $\bar{\sigma}_{\beta}^{\mathrm{SM}}$ respectively. The differential neutrino and antineutrino fluxes in the SM limit are

$$
\Phi^{\mathrm{SM}}=\frac{N_{\mu}}{4 \pi L^{2}} \frac{m_{\mu} E_{\nu}^{2}\left(4 E_{\nu}-3 m_{\mu}\right)}{24 \pi^{3} v^{4}}+\mathcal{O}\left(m_{e}\right), \quad \bar{\Phi}^{\mathrm{SM}}=\frac{N_{\mu}}{4 \pi L^{2}} \frac{m_{\mu} E_{\bar{\nu}}^{2}\left(m_{\mu}-2 E_{\bar{\nu}}\right)}{4 \pi^{3} v^{4}}+\mathcal{O}\left(m_{e}\right),
$$

where $N_{\mu}$ are the number of muons. Note that the two are different functions of the (anti)neutrino energy, which is due to the structure of the muon decay matrix element. The production coefficients are given by

$$
\begin{array}{lll}
p_{R L}=p_{L R}=-\frac{3 m_{e}}{3 m_{\mu}-4 E_{\nu}}+\mathcal{O}\left(m_{e}^{2}\right), & p_{R R}=\frac{6 m_{\mu}-12 E_{\nu}}{3 m_{\mu}-4 E_{\nu}}+\mathcal{O}\left(m_{e}\right) . \\
\bar{p}_{R L}=\bar{p}_{L R}=-\frac{m_{e}}{2 m_{\mu}-4 E_{\bar{\nu}}}, & \bar{p}_{R R}=\frac{3 m_{\mu}-4 E_{\bar{\nu}}}{6 m_{\mu}-12 E_{\bar{\nu}}}+\mathcal{O}\left(m_{e}\right) .
\end{array}
$$

and $p_{L L}=\bar{p}_{L L}=1$. Note that $p_{R L}$ and $\bar{p}_{R L}$, which control the linear effects proportional to the $\rho_{R}$ Wilson coefficients, are suppressed by $m_{e} / m_{\mu} \approx 0.005$. This makes the quadratic

\footnotetext{
${ }^{1}$ For leptonic tau decays the discussion is completely analogous, up to a trivial change of indices.

${ }^{2}$ The $R_{\mu \beta}$ expression including NP in detection is simply obtained replacing the SM detection piece $U_{\beta k} U_{\beta l}^{*}$ by the BSM detection piece shown in the second line of eq. (3.9), and likewise for $\bar{R}_{e \beta}$ with trivial changes.
} 
terms dominant unless $\rho_{R}$ are strongly suppressed, below $\mathcal{O}(1 \%)$, in which case they are probably too small to be observable in current experiments anyway. This fact amplifies the need for a correct treatment of quadratic terms, as ensured by our QFT formalism.

To discuss whether the above-given QFT result can be matched to the QM-NSI formalism one needs to specify how the latter describes (anti)neutrino production from muon decay $[6,44,45]$. Works carried out within the "non-unitary mixing matrix" setup [6] introduce in eq. (2.5) an additional normalization factor, $\left(N_{\delta}^{s}\right)^{2}$, associated to the (anti)neutrino $\nu_{\delta}$ that is emitted and not detected. In the $R_{\mu \beta}$ case discussed above, eq. (2.5) would be replaced by

$$
R_{\mu \beta}^{\mathrm{QM}}=\Phi_{\mu}^{\mathrm{SM}} \sigma_{\beta}^{\mathrm{SM}} P_{\mu \beta}^{\mathrm{QM}}\left(N_{e}^{s} N_{\mu}^{s} N_{\beta}^{d}\right)^{2} .
$$

The expression for the QM oscillation probability is not changed, and thus the additional normalization factor, $\left(N_{e}^{s}\right)^{2}$, does not cancel in the observable rate. One can check that the QFT rate in eq. (6.2) does reproduce such result at all orders only if the effective interactions are $V$ - $A$ and can be factorized as follows

$$
\delta_{\alpha a} \delta_{\beta b}+\left[\rho_{L}\right]_{a \alpha \beta b}=\left(\delta_{\alpha a}+\omega_{a \alpha}^{*}\right)\left(\delta_{\beta b}+\omega_{b \beta}\right) .
$$

In such a case, the matching is given by $\epsilon^{s}=\omega$. Such factorization holds in particular (but not only) when the BSM low-energy 4-lepton interaction is generated by modifications of the coupling of the $W$ boson to leptons, as e.g. in the "non-unitary mixing matrix" setup. However, if the $V$ - $A$ Wilson coefficient does not satisfy eq. (6.7), or if other interactions are present, the QM-NSI prescription in eq. (6.6) fails, even at linear order. That is, there is no matching between $\epsilon^{s}$ and $\rho_{X}$ such that eq. (6.6) is recovered from the QFT result in eq. (6.2).

One can formulate the QM-NSI approach in a different form, e.g. using eq. (2.5) without any modification

$$
R_{\mu \beta}^{\mathrm{QM}}=\Phi_{\mu}^{\mathrm{SM}} \sigma_{\beta}^{\mathrm{SM}} P_{\alpha \beta}^{\mathrm{QM}}\left(N_{\mu}^{s} N_{\beta}^{d}\right)^{2} .
$$

In that case it is possible to find a linear matching valid in general, namely

$$
\epsilon_{\mu \alpha}^{s}=\sum_{X} p_{X L}\left[\rho_{X}\right]_{e e \alpha \mu}, \quad \epsilon_{e \alpha}^{s}=\sum_{X} \bar{p}_{X L}\left[\rho_{X}\right]_{e \alpha \mu \mu} .
$$

However, this approach comes at the cost of (i) not being able to describe the relevant case of eq. (6.7) at all orders; and (ii) having process-dependent coefficients even in the $V$ - $A$ case.

All in all, we conclude that (anti)neutrino production through muon decay presents additional features with respect to the production through a semileptonic process. As a result, the limitations of the QM-NSI approach are even more severe. In particular we see that a generic $V-A$ interaction cannot be described exactly through the QM-NSI formalism.

In closing we note that the relation between the parameter $v$ and the Fermi constant $G_{F}$ becomes more complicated in the presence of the effective interactions in eq. (6.1):

$$
G_{F}^{2}=\frac{1}{2 v^{4}}\left[1+2 \operatorname{Re}\left[\rho_{L}\right]_{e e \mu \mu}+\sum_{\alpha \beta}\left(\left[\rho_{L}\right]_{e \alpha \beta \mu}\left[\rho_{L}\right]_{e \alpha \beta \mu}^{*}+\left[\rho_{R}\right]_{e \alpha \beta \mu}\left[\rho_{R}\right]_{e \alpha \beta \mu}^{*}\right)\right]+\mathcal{O}\left(m_{e}\right) .
$$

This is because the same Wilson coefficients that affect oscillations of neutrinos from muon decay also affect the muon lifetime, from which $G_{F}$ is determined experimentally. This has 
to be taken into account in order to derive consistent constraints on $\rho_{X}$ if absolute rates are used in the oscillation analysis. If on the other hand only ratios of rates at different distance $L$ are used, then $v$ cancels out in the observables, and this subtlety is irrelevant.

\section{Discussions and conclusions}

We close with several comments on the results derived above:

1. In this paper we only discussed charged-current NSI and assumed the absence of matter effects in propagation. The neutral-current NSI other than the matter effects can also be correctly described by QFT expressions analogous to eqs. (2.1)-(2.3), and they are relevant e.g. if neutrinos are detected via coherent scattering on nuclei. To include NSI entering via the matter effects one would need to modify the neutrino propagator in the derivation in appendix A starting from eq. (A.6). We leave this for future work.

2. It is worth stressing that charged-current NSI modify not only the flux and crosssection in eq. (2.2), but also the oscillation probability in vacuum. The latter follows directly from eq. (2.3), which depends on the production and detection amplitudes. Generically, that dependence does not cancel between the numerator and denominator in eq. (2.3).

3. The observable in eq. (2.1) may depend on NP in two distinct ways. One is direct, e.g. through a dependence of the production and detection amplitudes $\mathcal{M}_{\alpha k}^{P, D}$ on the NP parameters $\epsilon_{X}$ of the Lagrangian in eq. (3.1). The other is indirect, due to NP "polluting" the observable used for determination of the SM parameters [46]. This is the case for the CKM parameter $V_{u d}$ in eq. (3.1). If NP is present, $\beta$ decay experiments determine a combination of $V_{u d}$ and $\left[\epsilon_{X}\right]_{e \beta}$ parameters, and in this case the value of $V_{u d}$ cannot be just taken from PDG. This indirect effect is ignored in most of the prior neutrino literature, even though it is of the same order as the direct effects, leading to incorrect results. For instance, indirect and direct effects generated by the coefficient $\left[\epsilon_{L}\right]_{e e}$ cancel at all orders, making this coefficient unobservable in oscillation experiments [32].

The main results of this paper are: i) The expression in eq. (3.9) for the event rate in neutrino oscillation experiments including nonstandard charged-current interactions described by the EFT Lagrangian in eq. (3.1); ii) The matching in eq. (3.11) and table 1, valid at the linear level in NP, between the EFT coefficients that describe the underlying interactions and the QM-NSI parameters; iii) The consistency condition in eq. (3.12) for that matching (and the simplified QM-NSI approach itself) to be valid to all orders in NP parameters.

Our results are particularly relevant for analyses of oscillation data when effects of non-SM-like physics (or equivalently $\epsilon^{s} \neq \epsilon^{d \dagger}$ ) are taken into account [7, 9, 11-13, 15-17]. We give a more fundamental meaning to the long list of existing analyses of oscillation data carried out within the traditional QM-NSI approach. Their discovery potential can now be 
consistently analyzed and compared, among themselves and together with non-oscillation probes that are sensitive to the same underlying physics. Even at the linear level in $\epsilon_{X}$ we do find important and measurable effects that are not captured by the standard NSI formalism. Namely, in the presence of non-SM-like interactions in the EFT Lagrangian, we find that the NSI parameters depend on the neutrino energy. This dependence, which has several phenomenological consequences has never been considered before within the standard NSI formalism.

\section{Acknowledgments}

We thank Joachim Kopp for valuable discussions. We also acknowledge the hospitality of LPT Orsay, CERN-TH, and Karlsruhe Institute of Technology, where this work was developed. MGA is supported by the Generalitat Valenciana (Spain) through the plan GenT program (CIDEGENT/2018/014). ZT is supported by Fundação de Amparo à Pesquisa do Estado de São Paulo (FAPESP) under contract 2018/21745-8. AF is partially supported by the European Union's Horizon 2020 research and innovation programme under the Marie Skłodowska-Curie grant agreements No 690575 and No 674896.

\section{A Oscillations in QFT}

In this appendix we derive the master formula in eq. (2.1) describing the number of neutrino events detected at a distance $L$ from the source, taking into account possible neutrino oscillations and nonstandard charged-current interactions. Our approach follows similar steps as ref. [25]. The two main differences are: 1) we allow for general non-SM chargedcurrent interactions in neutrino production and detection, and 2) we work with timeindependent packets for the source and target particles, which greatly simplifies further mathematical transformations. Of course, the source is necessarily unstable, hence the latter assumption will lead to one subtlety in the derivation below.

We consider an experimental setup where neutrinos are produced in a process $A_{x} \rightarrow$ $X_{\alpha} \nu$, and detected via the process $\nu B_{y} \rightarrow Y_{\beta}$. Here $X_{\alpha}$ and $Y_{\beta}$ are $n_{x^{-}}$and $n_{y^{-b o d y} \text { final }}$ states $\left(n_{i} \geq 1\right)$. The indices $\alpha$ and $\beta$ indicate that these states contain charged lepton $\ell_{\alpha}$ and $\ell_{\beta}$ respectively, but otherwise their precise identity is irrelevant for this discussion. $A_{x}$ and $B_{y}$ are both one-body particle states localized in the coordinate space, describing the neutrino source (e.g. a beta-decaying nucleus in a reactor) and target (e.g. a proton in a detector). We will work in the time-independent approximation where the states $A_{x}$ and $B_{y}$ are represented by wave-packets of scattering in-states which do not change in time. We parametrize them as

$$
\left|A_{x}\right\rangle=\int \frac{d^{3} p_{A}}{\sqrt{2 E_{A}}(2 \pi)^{3}} \phi_{x}\left(p_{A}\right)\left|\vec{p}_{A}\right\rangle_{\mathrm{in}}, \quad\left|B_{y}\right\rangle=\int \frac{d^{3} p_{B}}{\sqrt{2 E_{B}}(2 \pi)^{3}} \phi_{y}\left(p_{B}\right)\left|\vec{p}_{B}\right\rangle_{\mathrm{in}},
$$

where $E_{j}=\sqrt{m_{j}^{2}+\left|p_{j}\right|^{2}}$, for $j=A, B,\left|\vec{p}_{j}\right\rangle_{\text {in }}$ are momentum eigenstates normalized as $\left\langle\vec{q}_{j} \mid \vec{p}_{j}\right\rangle=(2 \pi)^{3} 2 E_{j} \delta^{3}\left(\vec{p}_{j}-\vec{q}_{j}\right)$, and the states are normalized as $\left\langle A_{x} \mid A_{x}\right\rangle=\left\langle B_{y} \mid B_{y}\right\rangle=1$. 
For simplicity we choose Gaussian wave packets for both states with the same spread $\sigma$ in the position space:

$$
\phi_{z}(p)=(2 \sigma \sqrt{\pi})^{3 / 2} \exp \left(-|\vec{p}|^{2} \sigma^{2} / 2+i \vec{p} \vec{z}\right) .
$$

The wave packet describes a particle at rest localized near $\vec{z}$ with the uncertainty of order $\sigma$.

The idea is to treat the neutrino production and detection together as a single process [25]:

$$
A_{x} B_{y} \rightarrow X_{\alpha} Y_{\beta}
$$

rather than consider the neutrino production and detection separately. In this approach, neutrino is merely an intermediate particle in the amplitude. The outgoing states are approximated by pure momentum eigenstates with the eigenvalues $\vec{k}_{i}, i=1 \ldots n$, where $n=n_{x}+n_{y}$ is the number of particles in the final states. We are interested in the transition probability for this process:

$$
N_{\alpha \beta}=\left|\left\langle X_{\alpha} Y_{\beta} \mid A_{x} B_{y}\right\rangle\right|^{2}=\left.\left.\Pi_{i}\left[\int \frac{d^{3} k_{i}}{(2 \pi)^{3} 2 E_{i}}\right]\right|_{\text {out }}\left\langle k_{1} k_{2} \ldots k_{n} \mid A_{x} B_{y}\right\rangle\right|^{2} .
$$

Plugging the wave packets for the initial states, and using out $\left\langle k_{1} k_{2} \ldots k_{n} \mid p_{A} p_{B}\right\rangle_{\text {in }}=$ $(2 \pi)^{4} \delta^{4}\left(p_{A}+p_{B}-\sum k_{i}\right) \mathcal{M}$ we obtain

$$
\begin{aligned}
N_{\alpha \beta}= & \frac{1}{(2 \pi)^{8}} \int \frac{d^{3} p_{A}}{\sqrt{2 E_{A}}} \frac{d^{3} p_{B}}{\sqrt{2 E_{B}}} \frac{d^{3} p_{A}^{\prime}}{\sqrt{2 E_{A}^{\prime}}} \frac{d^{3} p_{B}^{\prime}}{\sqrt{2 E_{B}^{\prime}}} \\
& \times \phi_{x}\left(p_{A}\right) \phi_{y}\left(p_{B}\right) \phi_{x}\left(p_{A}^{\prime}\right)^{*} \phi_{y}\left(p_{B}^{\prime}\right)^{*} \delta^{4}\left(p_{A}^{\prime}+p_{B}^{\prime}-p_{A}-p_{B}\right) d \Pi_{n} \mathcal{M} \overline{\mathcal{M}}^{\prime},
\end{aligned}
$$

where $d \Pi_{n}=(2 \pi)^{4} \delta^{4}\left(p_{A}+p_{B}-\sum k_{i}\right) \Pi_{i} \frac{d^{3} k_{i}}{(2 \pi)^{3} 2 E_{i}}$ is the n-body phase space for the final-state particles, and $\mathcal{M} \equiv \mathcal{M}\left(p_{A} p_{B} \rightarrow k_{1} \ldots k_{n}\right), \mathcal{M}^{\prime} \equiv \mathcal{M}\left(p_{A}^{\prime} p_{B}^{\prime} \rightarrow k_{1} \ldots k_{n}\right)$ are the usual amplitudes calculated by Feynman diagrams. Tacitly, $N_{\alpha \beta}$ involves sum/average over all nonobserved degrees of freedom, such as polarizations of the initial- and final-state particles.

Up to this point, we followed the classic derivation of the cross section formula, see e.g. ref. [47]. What distinguishes the case at hand is the particular choice of the initial states $\left\langle A_{x}\right|,\left\langle B_{y}\right|$ describing two spatially separated particles (rather than head-on beams as in the cross section case). Furthermore, the amplitude for this process is dominated by the kinematic region where $q \equiv p_{A}-p_{X}=p_{Y}-p_{B}$ is close to the neutrino mass shell, $q^{2} \approx m_{k}^{2}$. In that region, unitarity requires the amplitude to factorize into the production, detection, and propagation parts:

$$
\mathcal{M}=\sum_{k} \frac{\mathcal{M}\left(p_{A} \rightarrow k_{X_{\alpha}} q_{\nu_{k}}\right) \mathcal{M}\left(q_{\nu_{k}} p_{B} \rightarrow k_{Y_{\beta}}\right)}{q^{2}-m_{k}^{2}+i \epsilon} \equiv \sum_{k} \frac{\mathcal{M}_{\alpha k}^{P} \mathcal{M}_{\beta k}^{D}}{q^{2}-m_{k}^{2}+i \epsilon},
$$

where the sum goes over all neutrino eigenstates, and the amplitudes in the numerator are evaluated for all particles on-shell, including the neutrinos. We can also factorize the phase space: $\int d \Pi_{n}=\int \frac{d q_{0}^{2}}{2 \pi} d \Pi_{P} d \Pi_{D}$, where the first factor is the $X+$ neutrino phase space, and the second factor is the $Y$ phase space. Next, it is convenient to isolate the phases in the 
wave packets by rewriting $\phi_{z}(p)=\tilde{\phi}(p) e^{i \overrightarrow{p z}}$. Finally, we trade one delta function for a time integral using $\delta\left(E_{A}+E_{B}-E_{A}^{\prime}-E_{B}^{\prime}\right)=\int_{-\infty}^{\infty} \frac{d t}{2 \pi} e^{i\left(E_{A}+E_{B}-E_{A}^{\prime}-E_{B}^{\prime}\right) t}$. This leads to

$$
\begin{aligned}
N_{\alpha \beta}= & \frac{1}{(2 \pi)^{9}} \int d t \frac{d^{3} p_{A}}{\sqrt{2 E_{A}}} \frac{d^{3} p_{B}}{\sqrt{2 E_{B}}} \frac{d^{3} p_{A}^{\prime}}{\sqrt{2 E_{A}^{\prime}}} \frac{d^{3} p_{B}^{\prime}}{\sqrt{2 E_{B}^{\prime}}} \tilde{\phi}\left(p_{A}\right) \tilde{\phi}\left(p_{B}\right) \tilde{\phi}\left(p_{A}^{\prime}\right) \tilde{\phi}\left(p_{B}^{\prime}\right) \\
& \times \delta^{3}\left(\vec{p}_{A}^{\prime}+\vec{p}_{B}^{\prime}-\vec{p}_{A}-\vec{p}_{B}\right) e^{i\left(E_{A}+E_{B}-E_{A}^{\prime}-E_{B}^{\prime}\right) t} e^{i\left(\vec{p}_{B}-\vec{p}_{B}^{\prime}\right) \vec{L}} \\
& \times \int \frac{d q_{0}^{2}}{2 \pi} d \Pi_{P} d \Pi_{D} \sum_{k l} \frac{\mathcal{M}_{\alpha k}^{P} \mathcal{M}_{\beta k}^{D}}{q^{2}-m_{k}^{2}+i \epsilon} \frac{\overline{\mathcal{M}}_{\alpha l}^{\prime} P}{\left(q+\overline{\mathcal{M}}_{\beta l}^{\prime} D\right.}
\end{aligned}
$$

where $\vec{L}=\vec{y}-\vec{x}$. The next step is to perform the $q_{0}^{2}$ integral, treating it as a contour integral:

$$
\begin{aligned}
N_{\alpha \beta}= & \frac{1}{(2 \pi)^{9}} \int d t \frac{d^{3} p_{A}}{\sqrt{2 E_{A}}} \frac{d^{3} p_{B}}{\sqrt{2 E_{B}}} \frac{d^{3} p_{A}^{\prime}}{\sqrt{2 E_{A}^{\prime}}} \frac{d^{3} p_{B}^{\prime}}{\sqrt{2 E_{B}^{\prime}}} \tilde{\phi}\left(p_{A}\right) \tilde{\phi}\left(p_{B}\right) \tilde{\phi}\left(p_{A}^{\prime}\right) \tilde{\phi}\left(p_{B}^{\prime}\right) \\
& \times \delta^{3}\left(\vec{p}_{A}^{\prime}+\vec{p}_{B}^{\prime}-\vec{p}_{A}-\vec{p}_{B}\right) e^{i\left(E_{A}+E_{B}-E_{A}^{\prime}-E_{B}^{\prime}\right) t} e^{i\left(\vec{p}_{B}-\vec{p}_{B}^{\prime}\right) \vec{L}_{d}} d \Pi_{P} d \Pi_{D} \\
& \times \sum_{k l} \frac{(-i) \mathcal{M}_{\alpha k}^{P} \mathcal{M}_{\beta k}^{D} \overline{\mathcal{M}}_{\alpha l}^{\prime P} \overline{\mathcal{M}}_{\beta l}^{\prime D}}{2 \sqrt{|\vec{q}|^{2}+m_{k}^{2}}\left(E_{B}-E_{B}^{\prime}\right)-2 \vec{q}\left(\vec{p}_{B}-\vec{p}_{B}^{\prime}\right)+\left(p_{B}-p_{B}^{\prime}\right)^{2}+\Delta m_{k l}^{2}-i \epsilon}
\end{aligned}
$$

where $\Delta m_{k l}^{2}=m_{k}^{2}-m_{l}^{2}$. Above, the amplitudes are now evaluated at $q_{0}=\sqrt{|\vec{q}|^{2}+m_{k}^{2}}$, that is for on-shell neutrinos.

At this point we introduce a number of approximations that are appropriate for the description of broad classes of real-life neutrino experiments:

1. The intermediate neutrinos are relativistic, hence in the production and detection amplitudes we can set $q_{0}=|\vec{q}|$. The dependence on the neutrino masses survives only via the $\Delta m_{k l}^{2}$ factor in eq. (A.8).

2. The wave packets are localized in an area much larger than the inverse mass of the source and target particles, $\sigma \gg m_{A, B}^{-1}$, such that $\left|\vec{p}_{A, B}\right| \sim \sigma^{-1} \ll m_{A, B}$. In the subsequent analysis we will only keep terms of $\mathcal{O}\left(\sigma^{-1}\right)$ and ignore those of $\mathcal{O}\left(\sigma^{-2}\right)$. In particular, we can approximate $E_{A} \approx m_{A}$ and $E_{B} \approx m_{B}$.

3. We ignore the dependence of the amplitudes on $\vec{p}_{j}$ or $\vec{p}_{j}^{\prime}$, from which it follows that $\mathcal{M}^{\prime}=\mathcal{M}$. Given the assumption in the previous point, this present assumption is safe whenever the amplitudes are dominated by a velocity-independent term.

With the above assumptions eq. (A.8) simplifies to

$$
\begin{aligned}
N_{\alpha \beta}= & \frac{\int d \Pi_{P} d \Pi_{D} \sum_{k l} \mathcal{M}_{\alpha k}^{P} \mathcal{M}_{\beta k}^{D} \overline{\mathcal{M}}_{\alpha l}^{P} \overline{\mathcal{M}}_{\beta l}^{D}}{(2 \pi)^{9}\left(2 m_{A}\right)\left(2 m_{B}\right)} \int d t d^{3} p_{A} d^{3} p_{B} d^{3} p_{A}^{\prime} d^{3} p_{B}^{\prime} \\
& \times \tilde{\phi}\left(p_{A}\right) \tilde{\phi}\left(p_{B}\right) \tilde{\phi}\left(p_{A}^{\prime}\right) \tilde{\phi}\left(p_{B}^{\prime}\right) \delta^{3}\left(\vec{p}_{A}^{\prime}+\vec{p}_{B}^{\prime}-\vec{p}_{A}-\vec{p}_{B}\right) \frac{(-i) e^{i\left(\vec{p}_{B}-\vec{p}_{B}^{\prime}\right) \vec{L}}}{\Delta m_{k l}^{2}-2 \vec{q}\left(\vec{p}_{B}-\vec{p}_{B}^{\prime}\right)-i \epsilon} .
\end{aligned}
$$

Due to our approximations, after replacing $e^{i\left(E_{A}+E_{B}-E_{A}^{\prime}-E_{B}^{\prime}\right) t} \approx 1$ nothing in the integrand depends on $t$ and thus the integral is infinite. This singularity could in fact be expected: due 
to using time-independent wave packets for the source $\left|A_{x}\right\rangle$ we tacitly integrate the rate of the $A_{x} B_{y} \rightarrow X Y$ process from $t=-\infty$ to $t=+\infty$. In a physical situation, however, $A_{x}$ is unstable, appears at a finite time $t_{0}$, and decays after a finite time $t_{0}+T$. Outside this window the process $A_{x} B_{y} \rightarrow X Y$ cannot occur. With this in mind, we drop the integration over $t$, and obtain the following result for the rate, that is the number of events per unit time:

$$
\begin{aligned}
\frac{d N_{\alpha \beta}}{d t}= & \frac{\int d \Pi_{P} d \Pi_{D} \sum_{k l} \mathcal{M}_{\alpha k}^{P} \mathcal{M}_{\beta k}^{D} \overline{\mathcal{M}}_{\alpha l}^{P} \overline{\mathcal{M}}_{\beta l}^{D}}{(2 \pi)^{9}\left(2 m_{A}\right)\left(2 m_{B}\right)} \int d^{3} p_{A} d^{3} p_{B} d^{3} p_{A}^{\prime} d^{3} p_{B}^{\prime} \\
& \times \tilde{\phi}\left(p_{A}\right) \tilde{\phi}\left(p_{B}\right) \tilde{\phi}\left(p_{A}^{\prime}\right) \tilde{\phi}\left(p_{B}^{\prime}\right) \delta^{3}\left(\vec{p}_{A}^{\prime}+\vec{p}_{B}^{\prime}-\vec{p}_{A}-\vec{p}_{B}\right) \frac{(-i) e^{i\left(\vec{p}_{B}-\vec{p}_{B}^{\prime}\right) \vec{L}}}{\Delta m_{k l}^{2}-2 \vec{q}\left(\vec{p}_{B}-\vec{p}_{B}^{\prime}\right)-i \epsilon} .
\end{aligned}
$$

Next, it is convenient to change the integration variables as $\vec{p}_{j}^{ \pm}=\vec{p}_{j} \pm \vec{p}_{j}^{\prime}$. Afterwards we can trivially perform the Gaussian integral over $d^{3} p_{A}^{+} d^{3} p_{B}^{+}$, and eliminate the integral over $d^{3} p_{A}^{-}$using the $\delta^{3}$. We also fix the coordinate frame such that $\vec{L}=(0,0, L)$, so that the $z$-axis connects the source and the target. This leads to

$$
\frac{d N_{\alpha \beta}}{d t}=\frac{\int d \Pi_{P} d \Pi_{D} \sum_{k l} \mathcal{M}_{\alpha k}^{P} \mathcal{M}_{\beta k}^{D} \overline{\mathcal{M}}_{\alpha l}^{P} \overline{\mathcal{M}}_{\beta l}^{D}}{(2 \pi)^{3}\left(2 m_{A}\right)\left(2 m_{B}\right)} \int d^{3} p \exp \left(-\frac{|\vec{p}|^{2} \sigma^{2}}{2}\right) \frac{(-i) e^{i L p_{z}}}{\Delta m_{k l}^{2}-2 \vec{q} \vec{p}-i \epsilon},
$$

where we simplified the notation: $\vec{p} \equiv \vec{p}_{B}^{-}=-\vec{p}_{A}^{-}$. In the integration over $p_{z}$, the principal value is suppressed by the rapidly oscillating $e^{i L p_{z}}$, and is neglected, which leaves the contribution from the pole at $p_{z}=\left(\Delta m_{k l}^{2}-2 q_{i} p_{i}\right) / 2 q_{z}$ :

$$
\begin{aligned}
\frac{d N_{\alpha \beta}}{d t}= & \frac{\int d \Pi_{P} d \Pi_{D} \sum_{k l} \mathcal{M}_{\alpha k}^{P} \mathcal{M}_{\beta k}^{D} \overline{\mathcal{M}}_{\alpha l}^{P} \overline{\mathcal{M}}_{\beta l}^{D}}{(2 \pi)^{3}\left(2 m_{A}\right)\left(2 m_{B}\right)} \exp \left(-\frac{\left(\Delta m_{k l}^{2}-2 q_{i} p_{i}\right)^{2} \sigma^{2}}{8 q_{z}^{2}}\right) \\
& \times \int d p_{x} d p_{y} \exp \left(-\frac{\left(\left|p_{x}\right|^{2}+\left|p_{y}\right|^{2}\right) \sigma^{2}}{2}\right) \exp \left(-i \frac{L q_{i} p_{i}}{q_{z}}+i \frac{L \Delta m_{k l}^{2}}{2 q_{z}}\right) \frac{\pi}{2 q_{z}}
\end{aligned}
$$

where $i=x, y$. Note that $q_{i}$ is the neutrino momentum in the "wrong", off-axis direction transverse to $\vec{L}$, thus $\left|q_{i}\right| \ll\left|q_{z}\right|$ as long as $L \gg \sigma$. Therefore we can drop $q_{i} / q_{z}$ factors everywhere, except when they are multiplied by $L$. Then we can trivially perform the Gaussian integral over $p_{x}$ and $p_{y}$, which leads to

$$
\begin{aligned}
\frac{d N_{\alpha \beta}}{d t}= & \frac{1}{32 \pi m_{A} m_{B} \sigma^{2}} \int d \Pi_{P} d \Pi_{D} \sum_{k l} \exp \left(-i \frac{L \Delta m_{k l}^{2}}{2 q_{z}}\right) \mathcal{M}_{\alpha k}^{P} \mathcal{M}_{\beta k}^{D} \overline{\mathcal{M}}_{\alpha l}^{P} \overline{\mathcal{M}}_{\beta l}^{D} \frac{1}{q_{z}} \\
& \times \exp \left(-\frac{\left(\Delta m_{k l}^{2}\right)^{2} \sigma^{2}}{8 q_{z}^{2}}\right) \exp \left(-\frac{\left(q_{x}^{2}+q_{y}^{2}\right) L^{2}}{2 q_{z}^{2} \sigma^{2}}\right) .
\end{aligned}
$$

The oscillatory factor $e^{-i 2 \pi L / L_{\mathrm{osc}}}$ appears here for the first time in this derivation, with the oscillation length $L_{\mathrm{osc}}=\frac{4 \pi q_{z}}{\Delta m_{k l}^{2}} \approx \frac{4 \pi E_{\nu}}{\Delta m_{k l}^{2}}$. In the QFT approach it arises because of interference between distinct neutrino mass eigenstates $k \neq l$ entering the propagators in eq. (A.7), which in turn is possible due to momentum uncertainty described by the initial state wave packets. The oscillations become suppressed by the factor $e^{-\Delta m_{k l}^{4} \sigma^{2} / 8 q_{z}^{2}}$ when the packet size becomes comparable to the oscillation length [48]. A condition for oscillations to be possible is

$$
\sigma \lesssim \frac{E_{\nu}}{\Delta m_{k l}^{2}} \sim L_{\mathrm{osc}}
$$


On the other hand, in our approach we do not find exponential suppression of the oscillations proportional to the distance $L$ travelled by the neutrino. The usual argument for this suppression [48, 49], due the decoherence of wave packets corresponding to different neutrino eigenstates traveling at different speeds, does not apply in the stationary situation considered here.

Due to the last exponential factor in eq. (A.13) the neutrino production angle $\theta \approx$ $\sqrt{q_{x}^{2}+q_{y}^{2}} / q_{z}$ must be such that $L \theta \lesssim \sigma$. This has a simple physical interpretation: the neutrino must hit the target within its position uncertainty described by the wave packet. Neutrinos emitted with $L \theta \gtrsim \sigma$ simply miss the target and do not contribute to the probability of the $A_{x} B_{y} \rightarrow X Y$ process. With this in mind, on the final transformation we trade $q_{z}=E_{\nu} \cos \theta \approx E_{\nu}$, and $q_{x}^{2}+q_{y}^{2}=E_{\nu}^{2} \sin ^{2} \theta \approx E_{\nu}^{2} \theta^{2}$. The production phase space contains the neutrino phase space $\frac{d^{3} q}{(2 \pi)^{3} 2 q_{0}}=\frac{E_{\nu} d E_{\nu} d \cos \theta d \phi}{16 \pi^{3}}$. One more assumption we need is that neutrinos are produced isotropically, that is $\mathcal{M}_{\alpha k}^{P} \overline{\mathcal{M}}_{\alpha l}^{P}$ integrate/summed over unobserved degrees of freedom is independent of the angular variables $\theta, \phi$. This assumption is satisfied in typical neutrino experiments where the source is unpolarized. The integral over $\theta$ can be evaluated order by order in $\sigma^{2} / L^{2}$, leading to

$$
\frac{d N_{\alpha \beta}}{d t d E_{\nu}}=\frac{1}{128 \pi^{3} L^{2} m_{A} m_{B}} \int d \Pi_{P-\nu} d \Pi_{D} \sum_{k l} e^{-i \frac{L \Delta m_{k l}^{2}}{2 E_{\nu}}} \mathcal{M}_{\alpha k}^{P} \mathcal{M}_{\beta k}^{D} \overline{\mathcal{M}}_{\alpha l}^{P} \overline{\mathcal{M}}_{\beta l}^{D} e^{\frac{-\left(\Delta m_{k l}^{2}\right)^{2} \sigma^{2}}{8 E_{\nu}^{2}}}
$$

where $d \Pi_{P}=d \Pi_{P-\nu} \frac{E_{\nu} d E_{\nu} d \cos \theta d \phi}{16 \pi^{3}}$, that is $d \Pi_{P-\nu}$ is the production phase space without the neutrino factor. Note the geometric $1 / L^{2}$ factor in front, which is of course expected intuitively. Mathematically, it appears due to integrating over the neutrino production angles in the phase space, where the contribution of off-axis neutrinos is exponentially suppressed and only the small cone $\theta \lesssim \sigma / L$ effectively contributes to the transition rate. The dependence on the size $\sigma$ of the initial wave packets has canceled out, except in the last exponential factor, which can be ignored in the limit $\sigma \Delta m_{k l}^{2} \ll E_{\nu}$. For an easier comparison with the expressions from the oscillation literature, it is convenient to put back the (now trivial) integration over the neutrino angular phase space variables $\theta$ and $\phi$ on the right-hand side of eq. (A.15):

$$
\frac{d N_{\alpha \beta}}{d t d E_{\nu}}=\frac{1}{32 \pi L^{2} m_{A} m_{B} E_{\nu}} \int d \Pi_{P^{\prime}} d \Pi_{D} \sum_{k l} e^{-i \frac{L \Delta m_{k l}^{2}}{2 E_{\nu}}} \mathcal{M}_{\alpha k}^{P} \mathcal{M}_{\beta k}^{D} \overline{\mathcal{M}}_{\alpha l}^{P} \overline{\mathcal{M}}_{\beta l}^{D} e^{\frac{-\left(\Delta m_{k l}^{2}\right)^{2} \sigma^{2}}{8 E_{\nu}^{2}}},
$$

where now $d \Pi_{P}=d \Pi_{P^{\prime}} d E_{\nu}$. In the limit $\sigma \Delta m_{k l}^{2} \ll E_{\nu}$, after multiplying the rate by the number of source and detector particles $N_{S, T}$ we obtain the master formula in eq. (2.1).

One can generalize eq. (A.16) to the case where the neutrino production is not isotropic $\left(\mathcal{M}_{\alpha k}^{P} \overline{\mathcal{M}}_{\alpha l}^{P}\right.$ depends on the neutrino production angles), and where the source is not at rest $\left(E_{A}>m_{A}\right)$. In the reference frame where the target is at rest, and in the limit $\sigma \Delta m_{k l}^{2} \ll E_{\nu}$, one finds

$$
\frac{d N_{\alpha \beta}}{d t d E_{\nu}}=\frac{E_{\nu}}{8 L^{2} E_{A} m_{B}} \sum_{k l} e^{-i \frac{L \Delta m_{k l}^{2}}{2 E_{\nu}}} \int d \Pi_{P^{\prime}} d \Pi_{D} \mathcal{M}_{\alpha k}^{P} \mathcal{M}_{\beta k}^{D} \overline{\mathcal{M}}_{\alpha l}^{P} \overline{\mathcal{M}}_{\beta l}^{D} \delta\left(q_{x}\right) \delta\left(q_{y}\right),
$$


where $q_{x, y}$ are the neutrino momenta perpendicular to $\vec{L}=(0,0, L)$, and we identify $q_{z}=$ $E_{\nu}$. Eq. (A.17) can be used, in particular, for neutrino produced via decays in flight, and for polarized neutrino production.

As a final comment, in this derivation we have assumed the absence of matter effects in propagation. The neutral-current NSI other than the matter effects can also be correctly described by QFT expressions analogous to eq. (A.16), and they are relevant e.g. if neutrinos are detected via coherent scattering on nuclei. To include NSI entering via the matter effects one would need to modify the neutrino propagator starting from eq. (A.6).

Open Access. This article is distributed under the terms of the Creative Commons Attribution License (CC-BY 4.0), which permits any use, distribution and reproduction in any medium, provided the original author(s) and source are credited.

\section{References}

[1] S.M. Bilenky and B. Pontecorvo, Lepton mixing and neutrino oscillations, Phys. Rept. 41 (1978) 225 [INSPIRE].

[2] P.F. de Salas, D.V. Forero, C.A. Ternes, M. Tortola and J.W.F. Valle, Status of neutrino oscillations 2018: $3 \sigma$ hint for normal mass ordering and improved CP sensitivity, Phys. Lett. $B \mathbf{7 8 2}$ (2018) 633 [arXiv:1708.01186] [INSPIRE].

[3] I. Esteban, M.C. Gonzalez-Garcia, A. Hernandez-Cabezudo, M. Maltoni and T. Schwetz, Global analysis of three-flavour neutrino oscillations: synergies and tensions in the determination of $\theta_{23}, \delta_{C P}$, and the mass ordering, JHEP 01 (2019) 106 [arXiv:1811.05487] [INSPIRE].

[4] S. Weinberg, Baryon and lepton nonconserving processes, Phys. Rev. Lett. 43 (1979) 1566 [INSPIRE].

[5] S. Bergmann, Y. Grossman and E. Nardi, Neutrino propagation in matter with general interactions, Phys. Rev. D 60 (1999) 093008 [hep-ph/9903517] [INSPIRE].

[6] S. Antusch, C. Biggio, E. Fernandez-Martinez, M.B. Gavela and J. Lopez-Pavon, Unitarity of the leptonic mixing matrix, JHEP 10 (2006) 084 [hep-ph/0607020] [INSPIRE].

[7] J. Kopp, M. Lindner, T. Ota and J. Sato, Non-standard neutrino interactions in reactor and superbeam experiments, Phys. Rev. D 77 (2008) 013007 [arXiv:0708.0152] [INSPIRE].

[8] A. Bolanos, O.G. Miranda, A. Palazzo, M.A. Tortola and J.W.F. Valle, Probing non-standard neutrino-electron interactions with solar and reactor neutrinos, Phys. Rev. D 79 (2009) 113012 [arXiv:0812.4417] [INSPIRE].

[9] T. Ohlsson and H. Zhang, Non-standard interaction effects at reactor neutrino experiments, Phys. Lett. B 671 (2009) 99 [arXiv:0809.4835] [INSPIRE].

[10] D. Delepine, V. Gonzalez Macias, S. Khalil and G. Lopez Castro, QFT results for neutrino oscillations and new physics, Phys. Rev. D 79 (2009) 093003 [arXiv:0901.1460] [INSPIRE].

[11] C. Biggio, M. Blennow and E. Fernandez-Martinez, General bounds on non-standard neutrino interactions, JHEP 08 (2009) 090 [arXiv:0907.0097] [INSPIRE].

[12] R. Leitner, M. Malinsky, B. Roskovec and H. Zhang, Non-standard antineutrino interactions at Daya Bay, JHEP 12 (2011) 001 [arXiv:1105.5580] [INSPIRE].

[13] T. Ohlsson, Status of non-standard neutrino interactions, Rept. Prog. Phys. 76 (2013) 044201 [arXiv: 1209.2710] [INSPIRE]. 
[14] A. Esmaili and A.Y. Smirnov, Probing non-standard interaction of neutrinos with IceCube and DeepCore, JHEP 06 (2013) 026 [arXiv:1304.1042] [INSPIRE].

[15] Y.-F. Li and Y.-L. Zhou, Shifts of neutrino oscillation parameters in reactor antineutrino experiments with non-standard interactions, Nucl. Phys. B 888 (2014) 137 [arXiv:1408.6301] [INSPIRE].

[16] S.K. Agarwalla, P. Bagchi, D.V. Forero and M. Tórtola, Probing non-standard interactions at Daya Bay, JHEP 07 (2015) 060 [arXiv:1412.1064] [inSPIRE].

[17] M. Blennow, S. Choubey, T. Ohlsson and S.K. Raut, Exploring source and detector non-standard neutrino interactions at ESSLSB, JHEP 09 (2015) 096 [arXiv:1507.02868] [INSPIRE].

[18] P. Coloma, M.C. Gonzalez-Garcia, M. Maltoni and T. Schwetz, COHERENT enlightenment of the neutrino dark side, Phys. Rev. D 96 (2017) 115007 [arXiv: 1708. 02899] [INSPIRE].

[19] Y. Farzan and M. Tortola, Neutrino oscillations and non-standard interactions, Front. in Phys. 6 (2018) 10 [arXiv:1710.09360] [INSPIRE].

[20] D. Choudhury, K. Ghosh and S. Niyogi, Probing nonstandard neutrino interactions at the LHC run II, Phys. Lett. B $\mathbf{7 8 4}$ (2018) 248 [arXiv: 1801.01513] [INSPIRE].

[21] J. Heeck, M. Lindner, W. Rodejohann and S. Vogl, Non-standard neutrino interactions and neutral gauge bosons, SciPost Phys. 6 (2019) 038 [arXiv:1812.04067] [INSPIRE].

[22] W. Altmannshofer, M. Tammaro and J. Zupan, Non-standard neutrino interactions and low energy experiments, JHEP 09 (2019) 083 [arXiv: 1812.02778] [INSPIRE].

[23] D. Aristizabal Sierra, V. De Romeri and N. Rojas, COHERENT analysis of neutrino generalized interactions, Phys. Rev. D 98 (2018) 075018 [arXiv: 1806.07424] [INSPIRE].

[24] I. Esteban, M.C. Gonzalez-Garcia, M. Maltoni, I. Martinez-Soler and J. Salvado, Updated constraints on non-standard interactions from global analysis of oscillation data, JHEP 08 (2018) 180 [arXiv : 1805.04530] [INSPIRE].

[25] C. Giunti, C.W. Kim, J.A. Lee and U.W. Lee, On the treatment of neutrino oscillations without resort to weak eigenstates, Phys. Rev. D 48 (1993) 4310 [hep-ph/9305276] [INSPIRE].

[26] E.K. Akhmedov and J. Kopp, Neutrino oscillations: quantum mechanics vs. quantum field theory, JHEP 04 (2010) 008 [Erratum ibid. 10 (2013) 052] [arXiv:1001.4815] [INSPIRE].

[27] A. Kobach, A.V. Manohar and J. McGreevy, Neutrino oscillation measurements computed in quantum field theory, Phys. Lett. B 783 (2018) 59 [arXiv:1711.07491] [inSPIRE].

[28] C. Giunti and C.W. Kim, Fundamentals of neutrino physics and astrophysics, Oxford University Press, Oxford, U.K. (2007) [InSPIRE].

[29] Y. Grossman, Nonstandard neutrino interactions and neutrino oscillation experiments, Phys. Lett. B 359 (1995) 141 [hep-ph/9507344] [INSPIRE].

[30] M.C. Gonzalez-Garcia, Y. Grossman, A. Gusso and Y. Nir, New CP-violation in neutrino oscillations, Phys. Rev. D 64 (2001) 096006 [hep-ph/0105159] [INSPIRE].

[31] V. Cirigliano, M. Gonzalez-Alonso and M.L. Graesser, Non-standard charged current interactions: beta decays versus the LHC, JHEP 02 (2013) 046 [arXiv: 1210 .4553] [INSPIRE].

[32] A. Falkowski, M. González-Alonso and Z. Tabrizi, Reactor neutrino oscillations as constraints on effective field theory, JHEP 05 (2019) 173 [arXiv:1901.04553] [INSPIRE]. 
[33] I. Bischer and W. Rodejohann, General neutrino interactions from an effective field theory perspective, Nucl. Phys. B 947 (2019) 114746 [arXiv:1905.08699] [INSPIRE].

[34] A.N. Khan, D.W. McKay and F. Tahir, Sensitivity of medium-baseline reactor neutrino mass-hierarchy experiments to nonstandard interactions, Phys. Rev. D 88 (2013) 113006 [arXiv:1305.4350] [INSPIRE].

[35] M. Blennow, P. Coloma, E. Fernandez-Martinez, J. Hernandez-Garcia and J. Lopez-Pavon, Non-unitarity, sterile neutrinos, and non-standard neutrino interactions, JHEP 04 (2017) 153 [arXiv: 1609.08637] [INSPIRE].

[36] Z.-Z. Xing, A full parametrization of the $6 \times 6$ flavor mixing matrix in the presence of three light or heavy sterile neutrinos, Phys. Rev. D 85 (2012) 013008 [arXiv:1110.0083] [INSPIRE].

[37] G.D. Cates, C.W. de Jager, S. Riordan and B. Wojtsekhowski, Flavor decomposition of the elastic nucleon electromagnetic form factors, Phys. Rev. Lett. 106 (2011) 252003 [arXiv: 1103.1808] [INSPIRE].

[38] M. González-Alonso and J. Martin Camalich, Isospin breaking in the nucleon mass and the sensitivity of $\beta$ decays to new physics, Phys. Rev. Lett. 112 (2014) 042501 [arXiv: 1309.4434] [INSPIRE].

[39] C.C. Chang et al., A per-cent-level determination of the nucleon axial coupling from quantum chromodynamics, Nature 558 (2018) 91 [arXiv: 1805.12130] [INSPIRE].

[40] R. Gupta, Y.-C. Jang, B. Yoon, H.-W. Lin, V. Cirigliano and T. Bhattacharya, Isovector charges of the nucleon from $2+1+1$-flavor lattice QCD, Phys. Rev. D 98 (2018) 034503 [arXiv: 1806. 09006] [INSPIRE].

[41] Flavour Lattice Averaging Group collaboration, FLAG review 2019: Flavour Lattice Averaging Group (FLAG), Eur. Phys. J. C 80 (2020) 113 [arXiv:1902.08191] [InSPIRE].

[42] J. Kopp, New phenomena in neutrino physics, Ph.D. thesis, Heidelberg U., Heidelberg, Germany (2009) [INSPIRE].

[43] P. Langacker and D. London, Lepton number violation and massless nonorthogonal neutrinos, Phys. Rev. D 38 (1988) 907 [INSPIRE].

[44] J. Tang and Y. Zhang, Study of nonstandard charged-current interactions at the MOMENT experiment, Phys. Rev. D 97 (2018) 035018 [arXiv:1705.09500] [InSPIRE].

[45] C. Soumya, M. Ghosh, S.K. Raut, N. Sinha and P. Mehta, Probing muonic charged current nonstandard interactions at decay-at-rest facilities in conjunction with T2HK, Phys. Rev. D 101 (2020) 055009 [arXiv:1911.05021] [INSPIRE].

[46] S. Descotes-Genon, A. Falkowski, M. Fedele, M. González-Alonso and J. Virto, The CKM parameters in the SMEFT, JHEP 05 (2019) 172 [arXiv: 1812.08163] [INSPIRE].

[47] M.E. Peskin and D.V. Schroeder, An introduction to quantum field theory, Addison-Wesley, Reading, MA, U.S.A. (1995).

[48] B. Kayser, On the quantum mechanics of neutrino oscillation, Phys. Rev. D 24 (1981) 110 [INSPIRE].

[49] S. Nussinov, Solar neutrinos and neutrino mixing, Phys. Lett. B 63 (1976) 201 [INSPIRE]. 